\title{
Redução do risco cardiovascular global através da continuidade de cuidados assistenciais prestados a uma população hipertensa pelo Médico de Família
}

Hiroshi Okai,* Mónica Marlene Correia Lopes**

\section{RESUMO}

Introdução: As doenças cardiovasculares são a principal causa de mortalidade em Portugal, representando 34\% das mortes ocorridas em 2005. A Direcção-Geral da Saúde recomenda a utilização da tabela derivada do projecto SCORE para o cálculo do risco cardiovascular global (RCVG), permitindo identificar os doentes com risco elevado que mais beneficiam com o controlo efectivo dos factores de risco cardiovascular (FRCV).

O Médico de Família encontra-se em posição privilegiada para actuar nos FRCV modificáveis da população hipertensa e reduzir o seu RCVG. Existe evidência diversa a demonstrar que a continuidade de cuidados resulta na melhoria dos resultados em diversas condições crónicas, como a hipertensão arterial, diabetes mellitus e asma.

Objectivos: Verificar se a continuidade de cuidados assistenciais prestados pelo Médico de Família aos hipertensos condicionaram: (1) redução do RCVG; (2) melhoria dos FRCV major; (3) maior redução da TA e do perfil lipídico nos hipertensos com RCVG inicial alto. Metodologia: Estudo longitudinal retrospectivo quasi-experimental. População-alvo: hipertensos diagnosticados até 2004 e frequentadores da consulta de hipertensão entre 2004 e 2007 (n=197). Variáveis estudadas: TA, perfil lipídico, tabagismo e RCVG. Resultados: Comparando as médias dos valores de RCVG obtidos com aplicação da tabela SCORE, obteve-se uma redução significativa de 19,2\% em 3 anos. Quanto aos FRCV, verificou-se significativamente: uma redução média de 9,3\% a nível da tensão arterial sistólica (TAS) e um aumento de 3,2\% na tensão arterial diastólica; uma redução média de $6,5 \%$ e $13,8 \%$ a nível do colesterol total (CT) e do colesterol-LDL (C-LDL); um aumento do colesterol-HDL e dos triglicerídeos de 5,4\% e 8,4\%, respectivamente; e uma redução de $50 \%$ no consumo tabágico. Os hipertensos inicialmente de alto risco obtiveram o triplo da redução ocorrida a nível da TAS e do CT e o dobro a nível do C-LDL em relação aos de baixo risco.

Discussão: Observou-se uma redução significativa do RCVG e uma melhoria dos FRCV major. Houve uma actuação adaptada aos níveis iniciais de risco, resultando em maior benefício para aqueles com alto risco inicial.

Palavras-Chave: Risco Cardiovascular Global; Continuidade de Cuidados; Médico de Família.

\section{INTRODUÇÃO}

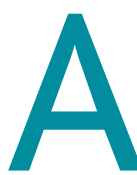

s doenças cardiovasculares, nomeadamente os acidentes vasculares cerebrais (AVC) e a doença isquémica do coração (DIC), são a principal causa de mortalidade em Portugal,

*Assistente de Medicina Geral e Familiar

**Aluna do $6^{\circ}$ Ano Médico - Mestrado Integrado de Medicina

Unidade de Saúde do Vale - Centro de Saúde de Santa Maria da Feira; Faculdade de Medicina da Universidade de Coimbra sendo considerada das mais elevadas na Europa e no Mundo. ${ }^{1}$ Estas doenças foram responsáveis por cerca de $34 \%$ das mortes ocorridas em 2005, contando-se, também, entre as principais causas de morbilidade, invalidez e potenciais anos de vida perdidos na população. ${ }^{2}$

Observa-se, no entanto, uma tendência decrescente a nível nacional e em todos os grupos etários, sendo que $44,3 \%$ destes óbitos correspondem a AVC e $23,5 \%$ a DIC (Figura 1). 


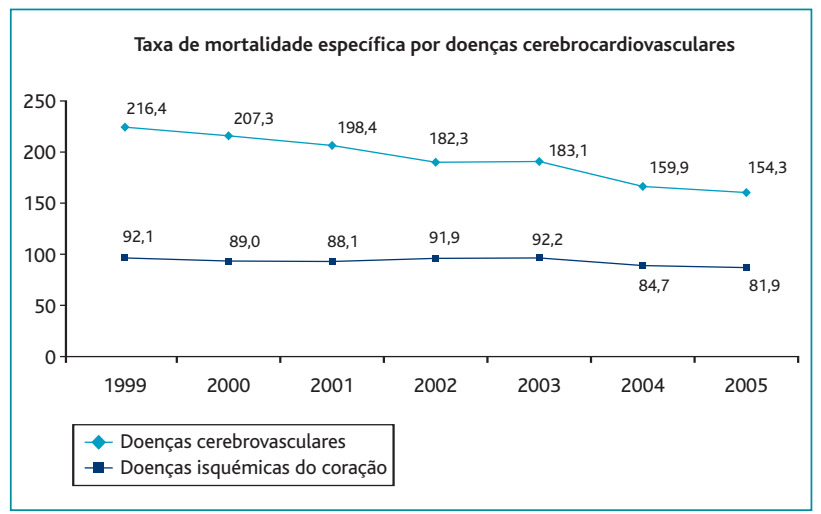

Figura 1. Evolução da taxa de mortalidade específica (\%000) por doenças cerebrovasculares e isquémicas do coração entre 1999 e 2005.

O cálculo do RCVG, como estimativa do sinergismo dos factores de risco individuais, permite não só identificar doentes com risco elevado, bem como modular a intensidade de intervenção terapêutica no controlo efectivo desses factores, motivar os doentes para uma adesão plena às medidas farmacológicas e modificadoras de estilos de vida, realçando o grau de risco, ganhos potenciais das intervenções propostas e a valorização da necessidade e efectividade dos tratamentos. ${ }^{3}$

Para determinar o RCVG, a Direcção-Geral de Saúde recomenda a utilização da tabela derivada do projecto SCORE (Systematic Coronary Risk Evaluation), desenvolvida pelo conjunto de Sociedades Científicas congéneres Europeias, com base em estudos observacionais prospectivos de populações europeias. ${ }^{3}$

Constituem limitações importantes para a utilização da escala de Framingham, o facto desta reflectir a realidade duma pequena população do nordeste americano, sobrestimando o risco de doença coronária nas populações de baixo risco do sul da Europa, que apresentam características e estilos de vida muito diferentes. ${ }^{4}$

Os benefícios das diversas intervenções terapêuticas dependem dos níveis iniciais do risco: quanto mais elevado, maior o benefício. Diversos pacientes com HTA não têm sido tratados com agressividade suficiente, nomeadamente aqueles com risco cardiovascular mais elevado. ${ }^{5,6}$

O RCVG avaliado pela tabela SCORE varia consoante os factores de risco: sexo, idade, tensão arterial (TA), colesterol total (CT) e hábitos tabágicos.

A prevalência da HTA em Portugal cifra-se em 42,1\%, sendo que apenas $39 \%$ dos doentes estão tratados e $11,2 \%$ controlados $(\mathrm{PA}<140 / 90 \mathrm{mmHg}){ }^{7}$

A mortalidade, por DIC e AVC, aumenta progressiva e linearmente a partir do nível tensional 115/75mmHg; por cada incremento de $20 \mathrm{mmHg}$ na TAS ou $10 \mathrm{mmHg}$ na TAD, ocorre uma duplicação da mortalidade para ambas as doenças. ${ }^{8}$

Parte considerável da população $(68,5 \%)$ apresenta risco moderado para doença cardiovascular (CT $\geq 190 \mathrm{mg} / \mathrm{dl}$ ) e cerca de $23,4 \%$ apresenta risco elevado (CT $\geq 240 \mathrm{mg} / \mathrm{dl})$. O valor de CT médio da população encontra-se nos $210 \mathrm{mg} / \mathrm{dl}$, todavia também possuem um valor médio elevado para C-HDL (52,3 mg/dl). ${ }^{9}$

A redução de $10 \%$ do CT plasmático condiciona redução de $25 \%$ na incidência de doença coronária após 5 anos, e redução do C-LDL de cerca de $40 \mathrm{mg} / \mathrm{dl}$ acompanha-se pela redução de doença coronária em $23 \%$, $19 \%$ de AVC e $21 \%$ de doenças cardiovasculares. ${ }^{10}$

Verifica-se ter havido um ligeiro decréscimo nas prevalências totais de consumo tabágico em Portugal Continental (1998 a 2005), na população com $\geq 15$ anos: de $20,5 \%$ para $20,2 \%$. Este consumo é, como em todo o mundo, das principais causas de morbi-mortalidade evitáveis. ${ }^{11,12}$

Existe evidência diversa que associa continuidade de cuidados a benefícios de saúde (melhoria da qualidade dos cuidados, nomeadamente patologias crónicas, cuidados preventivos, redução da hospitalização, eficiência)..$^{13,14}$

A lista de utentes objecto do estudo esteve cerca de três anos sem Médico de Família atribuído, sendo então assistida por diversos médicos em consultas de reforço.

A continuidade de cuidados assistenciais prestados ao longo de 3 anos desde 2004 pelo Médico de Família ao grupo de risco/população hipertensa da sua lista, pode resultar na melhoria dos FRCV modificáveis e, consequentemente, do RCVG.

\section{OBJECTIVOS}

Verificar se a continuidade de cuidados assistenciais prestados pelo Médico de Família aos hipertensos condicionaram após 3 anos: (1) redução do RCVG; (2) melhoria dos FRCV major modificáveis (TA, perfil lipídi- 
co, tabagismo); (3) maior redução da TA e do perfil lipídico nos hipertensos com RCVG inicial alto.

\section{METODOLOGIA}

Tipo

Estudo longitudinal retrospectivo quasi-experimental.

\section{População-alvo}

A população hipertensa representa um grupo de risco que necessita de cuidados assistenciais regulares. Em 2004, estavam identificados 251 hipertensos na lista de utentes do Médico de Família. Este exerce funções na Unidade de Saúde do Vale (Centro de Saúde de Santa Maria da Feira), sita numa localidade de características rurais, distanciada a cerca de $10 \mathrm{~km}$ dos centros urbanos de Santa Maria da Feira e S. João da Madeira.

No presente estudo, foram definidos os seguintes critérios para selecção da população-alvo:

- Critérios de inclusão:

(1) Utentes hipertensos diagnosticados até 2004; (2) Frequentadores da consulta do Médico de Família em consultas de hipertensão pelo menos 1 vez por ano.

- Critérios de exclusão:

(1) Não utilizadores ou sem consultas presenciais pelo menos 1 vez por ano; (2) Grávidas; (3) Falecimento durante o período de avaliação.

Após aplicação dos critérios de selecção, obteve-se a dimensão da população de 197 hipertensos, objecto da avaliação do estudo, cujas características iniciais encontram-se descritas no Quadro I.

\section{Variáveis}

(1) Tensão arterial: tensão arterial sistólica (TAS), tensão arterial diastólica (TAD)

Valores da TA registados na $1^{\text {a }}$ consulta de hipertensão realizada de 2004 a 2007 (condições prévias de medição da TA - em repouso, 5 minutos após sentar, valor obtido com 1 medição ou média de 2 medições, com esfigmomanómetro aneróide e desde Fevereiro de 2007 com esfigmomanómetro automático oscilométrico).

- Definição de TA controlada: utilizou-se como critério de controlo tensional a TA $<140 / 90 \mathrm{~mm} \mathrm{Hg}$ (critérios nacional e internacionalmente aceites) ${ }^{15,16}$

(2) Perfil lipídico: CT, C-HDL, TG, C-LDL.

Valores do $1^{\circ}$ perfil lipídico registados nos anos de 2004 e 2007.

\begin{tabular}{|c|c|}
\hline \multicolumn{2}{|l|}{$\begin{array}{l}\text { QUADRO I. Características iniciais da população } \\
\text { hipertensa em estudo }\end{array}$} \\
\hline Características $(n=197)$ & Valor \\
\hline Sexo - $n^{\circ}$ mulheres (\%) & $123(62,4)$ \\
\hline Idade - anos* & $67,2(10,8)$ \\
\hline Duração da hipertensão - anos* & $4,3(4,7)$ \\
\hline \multicolumn{2}{|l|}{ Número de medicamentos hipotensores $-\mathrm{n}^{\circ}(\%)$} \\
\hline 0 & $35(17,8)$ \\
\hline 1 & $86(43,7)$ \\
\hline 2 & $65(33,0)$ \\
\hline$\geq 3$ & $11(5,6)$ \\
\hline \multicolumn{2}{|l|}{ Condições coexistentes seleccionadas $-\mathrm{n}^{\circ}(\%)$} \\
\hline Diabetes mellitus & $44(22,3)$ \\
\hline Dislipidemia & $107(54,3)$ \\
\hline Doença arterial coronária & $12(6,1)$ \\
\hline Doença cerebrovascular & $25(12,7)$ \\
\hline
\end{tabular}

*média \pm desvio-padrão

- Definição de perfil lipídico controlado: CT inferior a $190 \mathrm{mg} / \mathrm{dl}$ e C-LDL inferior a $115 \mathrm{mg} / \mathrm{dl} .^{6,9}$

\section{(3) Hábitos tabágicos}

Consumo tabágico registado na $1^{\text {a }}$ consulta de hipertensão realizada em 2004 e 2007 - operacionalizada para estado de fumador actual (consumo de pelo menos 1 cigarro por dia) ou não fumador.

(4) Risco cardiovascular global: SCORE

Valores do RCVG utilizando a tabela SCORE em 2004 e 2007 com base nos dados obtidos nas consultas inicial e final e perfis lipídicos.

Por uma questão de precisão e uniformização de critérios, efectuaram-se cálculos do risco cardiovascular global através do programa informático HeartScore ${ }^{\circledast}$ disponível no sítio electrónico da European Society of Cardiology. ${ }^{17}$

Existem situações potenciadoras de RCVG superior ao indicado na tabela, nas quais foram aplicadas coeficientes correctores ao valor calculado no SCORE, nomeadamente quando o indivíduo tinha diabetes (multiplicando por 2 o risco calculado nos homens e por 4 nas mulheres) ou tinha doença cerebrovascular (multiplicando por 1,5 o risco calculado). ${ }^{3}$

A idade pode constituir um factor importante na variação do risco cardiovascular; foi efectuada uma correcção para o cálculo do risco na consulta final, utili- 


\section{QUADRO II. Comparação entre médias do SCORE inicial e final

\begin{tabular}{l|c|c|c} 
SCORE & Inicial & Final & $\boldsymbol{P}$ \\
\hline Média $( \pm d p)$ & $5,00( \pm 4,15)$ & $4,04( \pm 3,93)$ & $<0,001$
\end{tabular}

Teste t Student para amostras emparelhadas; $n=197$

zando a idade que o hipertenso tinha na consulta inicial, de modo a podermos aferir a eventual redução do risco associado às mudanças nos factores de risco modificáveis, sem influência da idade.

- Definição de alto risco: Com base no risco de morte cardiovascular aos 10 anos, considera-se como categoria de alto risco, susceptível de medidas eficazes de prevenção farmacológica, risco absoluto maior ou igual a 5\%. ${ }^{3}$

\section{Duração}

A avaliação decorreu, para cada utente, entre a $1^{\text {a }}$ consulta de hipertensão de 2004 (consulta inicial) e a $1^{\text {a }}$ consulta de hipertensão de 2007 (consulta final). O tempo médio do estudo foi de 2,9 ( $\pm 0,3)$ anos (mín 2,1; máx 3,7).

\section{Colheita de informação}

A colheita de dados foi efectuada através da consulta dos registos clínicos dos utentes pertencentes ao ficheiro do Médico de Família, e posteriormente introduzidos numa matriz de dados electrónica (Excel $\left.{ }^{\circledR} 2003\right)^{18}$. A listagem dos hipertensos foi fornecida pelo Serviço de Informática da Sub-região de Saúde de Aveiro, a partir da codificação efectuada no programa informático SAM (Software de Apoio ao Médico).

\section{Tratamento de dados}

O teste de McNemar foi aplicado no estudo da associação entre variáveis categóricas de grupos emparelhados (antes e depois).

O teste $t$ de Student para amostras emparelhadas foi utilizado para a associação entre variáveis quantitativas (comparação da média no mesmo grupo mas em momentos diferentes).

Todos os testes estatísticos foram efectuados para um nível de significância de $95 \%$.

Foi utilizado o programa estatístico SPSS ${ }^{\circledR}$ versão 10.0. ${ }^{19}$

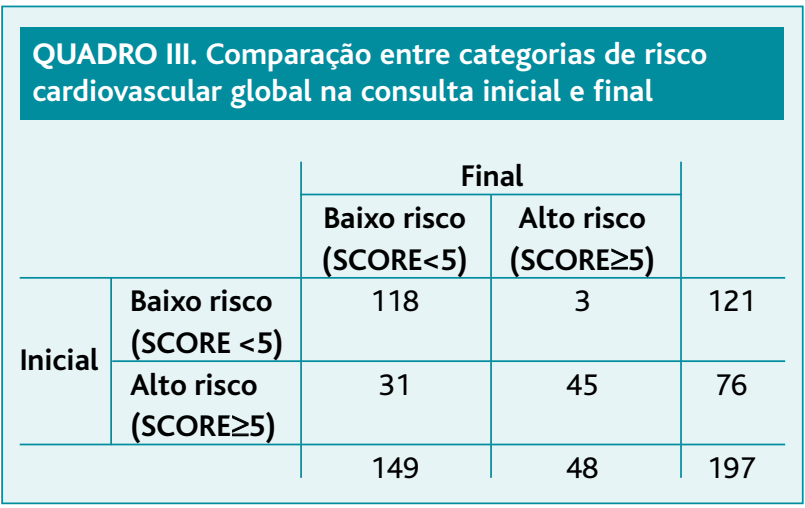

Teste de McNemar; $p<0,001$

\section{RESULTADOS}

$1^{\circ}$ Objectivo: Redução do risco cardiovascular global (SCORE)

Verificou-se que a continuidade dos cuidados assistenciais prestados pelo Médico de Família ao longo de 3 anos à população de hipertensos condiciona redução significativa do seu RCVG.

Na comparação entre as médias dos valores de risco cardiovascular global obtidos com a aplicação da tabela SCORE (posteriormente corrigidas), obteve-se uma redução significativa de 19,2\% $(p<0,001)$ (Quadro II).

Na comparação entre categorias de risco, verificou-se que o número de hipertensos considerados de alto risco (SCORE $\geq 5$ ) foi reduzido em $60,3 \%$ (de 76 para 48 ), enquanto que, inversamente, os hipertensos de baixo risco $(\mathrm{SCORE}<5)$ aumentaram $81,2 \%$ (de 121 para 149 ) $(p<0,001)$.

Esta diferença foi possível porque 31 hipertensos considerados de alto risco na consulta inicial evoluíram para a categoria de baixo risco na consulta final, enquanto apenas 3 hipertensos inicialmente de baixo risco regrediram para a categoria de alto risco (Quadro III).

\section{$2^{\circ}$ Objectivo: Melhoria dos factores de risco} cardiovascular major

\section{Tensão arterial}

Quanto à TA, verificou-se uma redução média significativa $(p<0,001)$ de cerca de $9,3 \%$ a nível da TAS, o que equivale a $-10,9 \mathrm{mmHg}$. Ao invés, houve um aumento médio de 2,5mmHg a nível da TAD, representando um acréscimo de 3,2\% $(p=0,008)$ (Quadro IV). 


\begin{tabular}{|c|c|c|c|}
\hline \multicolumn{4}{|c|}{$\begin{array}{l}\text { QUADRO IV. Evolução dos FRCV major entre a } \\
\text { consulta inicial e final }\end{array}$} \\
\hline & Inicial & Final & $P$ \\
\hline TAS & $153,7( \pm 21,4)$ & $142,8( \pm 17,6)$ & $<0,001$ \\
\hline TAD & $78,5( \pm 16,0)$ & $81( \pm 11,8)$ & 0,008 \\
\hline CT & $208,8( \pm 42,0)$ & $195,3( \pm 33,1)$ & $<0,001$ \\
\hline $\mathrm{C}-\mathrm{HDL}$ & $53,6( \pm 12,8)$ & $56,5( \pm 13,7)$ & $<0,001$ \\
\hline TG & $112,6( \pm 52,4)$ & $122,0( \pm 54,8)$ & $<0,001$ \\
\hline C-LDL & $132,7( \pm 37,9)$ & $114,4( \pm 29,1)$ & $<0,001$ \\
\hline
\end{tabular}

Teste $\mathrm{t}$ de Student para amostras emparelhadas; $\mathrm{n}=197$

\section{HTA controlada}

Quanto ao grau de controlo tensional, verificou-se um aumento significativo $(p<0,001)$ de $133,3 \%$ de hipertensos com TA $<140 / 90 \mathrm{mmHg}$ no final do estudo, o que representa uma evolução de 36 para 84 hipertensos controlados (Quadro V).

\section{Perfil lipídico}

Obteve-se uma melhoria global do perfil lipídico, à excepção dos TG. Quanto ao CT e ao C-LDL, houve uma redução média significativa de $13,5 \mathrm{mg} / \mathrm{dl}$ (6,5\%; $p<0,001)$ e $18,3 \mathrm{mg} / \mathrm{dl}(13,8 \% ; p<0,001)$, respectivamente. Inversamente, houve um aumento médio significativo do C-HDL e TG de 2,9mg/dl $(5,4 \% ; p<0,001)$ e $9,4 \mathrm{mg} / \mathrm{dl}(8,4 \% ; p<0,001)$, respectivamente (Quadro IV).

\section{Dislipidémicos controlados}

Relativamente ao grau de controlo da dislipidemia, verificou-se: (1) um aumento de $24,7 \%$ embora estatisticamente não significativo ( $p=0,057)$ de hipertensos com $\mathrm{CT}<190 \mathrm{mg} / \mathrm{dl}$ (de 73 para 91); e (2) um aumento significativo $(p<0,001)$ de $54,4 \%$ de hipertensos com a C-LDL $<115 \mathrm{mg} / \mathrm{dl}$ (de 68 para 105) (Quadro V).

\section{Tabagismo}

Dos dois hipertensos que eram fumadores no início do estudo, um manteve os seus hábitos tabágicos ao fim de 3 anos, representando uma redução de $50 \%(p=0,01)$ (Quadro V).

\section{$3^{\circ}$ Objectivo: Melhoria dos FRCV nos hipertensos} de alto vs baixo risco

Verificou-se que os hipertensos inicialmente de alto ris-

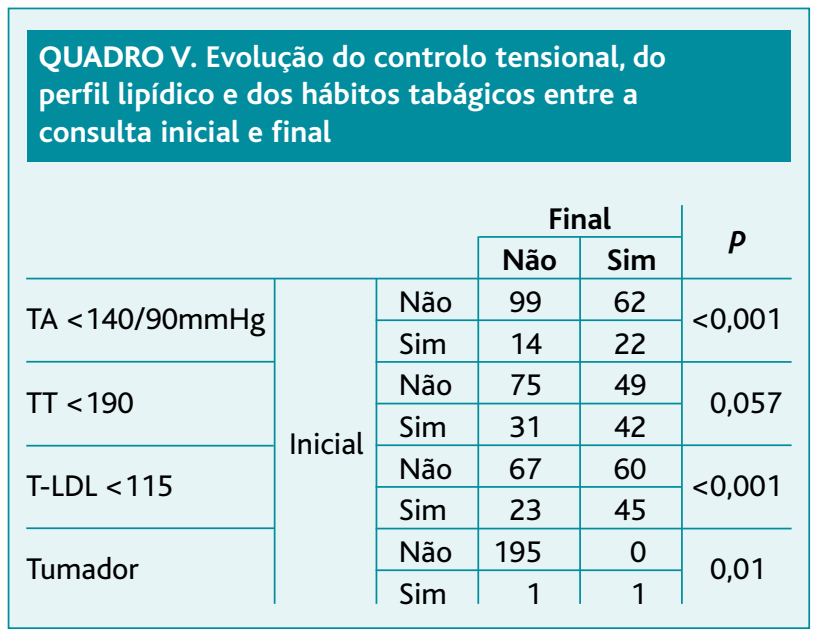

Teste de McNemar; $\mathrm{n}=197$

co obtiveram uma redução mais acentuada da TAS, de cerca de $18,3 \mathrm{mmHg}$, o que representa quase o triplo do valor alcançado pelos hipertensos de baixo risco $(6,2 \mathrm{mmHg} ; p=0,004)$. O aumento na TAD não foi significativo. (Quadro VI)

Verificou-se ainda que os hipertensos inicialmente de alto risco obtiveram uma redução significativa a nível do CT e C-LDL respectivamente em cerca de $24,1 \mathrm{mg} / \mathrm{dl}(11,2 \% ; p<0,001)$ e $25,6 \mathrm{mg} / \mathrm{dl}(18,4 \%$; $p<0,001)$, enquanto que os de baixo risco obtiveram uma redução média de $7,0 \mathrm{mg} / \mathrm{dl}(3,4 \% ; p=0,05)$ e 13,8mg/dl (10,7\%; $p<0,001)$ (Quadro VI).

\section{DISCUSSÃO}

Um sistema de saúde baseado nos cuidados de saúde primários constitui um factor fundamental na prevenção das doenças cardiovasculares. A população do presente estudo pode representar o universo de utentes hipertensos (intrinsecamente com elevado risco cardiovascular associado) sem Médico de Família atribuído que, caso beneficiassem do acesso adequado e continuado dos cuidados assistenciais prestados pelo mesmo Médico de Família, poderiam obter uma redução do seu RCVG e melhoria a nível dos FRCV major modificáveis.

Além disso, os hipertensos de alto risco inicial obtiveram cerca do triplo da redução dos valores tensionais e do CT e o dobro da redução do C-LDL em relação aos de baixo risco, facto que indica uma intervenção mais 


\begin{tabular}{|c|c|c|c|c|c|}
\hline \multicolumn{6}{|c|}{$\begin{array}{l}\text { QUADRO VI. Evolução da TA, do CT e C-LDL dos hipertensos de alto e baixo risco entre } \\
\text { a consulta inicial e final }\end{array}$} \\
\hline & & Inicial & Final & $\Delta$ & $P$ \\
\hline \multirow{4}{*}{$\begin{array}{l}\text { Alto risco } \\
(n=76)\end{array}$} & TAS & $164,8( \pm 21,4)$ & $146,6( \pm 16,9)$ & $18,3( \pm 25,4)$ & $<0,001$ \\
\hline & TAD & $78,7( \pm 17,0)$ & $80,7( \pm 10,4)$ & $-2,0( \pm 18,9)$ & 0,356 \\
\hline & $\mathrm{CT}$ & $215,9( \pm 41,8)$ & $191,9( \pm 28,4)$ & $24,1( \pm 44,8)$ & $<0,001$ \\
\hline & C-LDL & $138,8( \pm 37,9)$ & $113,2( \pm 26,2)$ & $25,6( \pm 41,9)$ & $<0,001$ \\
\hline \multirow{4}{*}{$\begin{array}{l}\text { Baixo risco } \\
(n=121)\end{array}$} & TAS & $146,7( \pm 18,3)$ & $140,5( \pm 17,6)$ & $6,2( \pm 23,2)$ & 0,004 \\
\hline & TAD & $78,4( \pm 15,5)$ & $82,6( \pm 12,6)$ & $-4,1( \pm 16,3)$ & 0,006 \\
\hline & CT & $204,4( \pm 41,7)$ & $197,4( \pm 35,6)$ & $7,0( \pm 38,9)$ & 0,05 \\
\hline & C-LDL & $128,8( \pm 37,6)$ & $115,1( \pm 30,9)$ & $13,8( \pm 36,9)$ & $<0,001$ \\
\hline
\end{tabular}

Teste $\mathrm{t}$ Student para amostras emparelhadas; $\mathrm{n}=197$

intensiva por parte do Médico de Família sobre esta sub-população de maior risco.

Existem diversos aspectos que podem condicionar a interpretação dos resultados; esta deve ser feita com cautela, considerando a sua validade externa e interna, possíveis vieses e variáveis de confundimento.

Os autores não encontraram outros trabalhos metodologicamente semelhantes, de modo a poderem comparar os resultados obtidos.

Quanto à validade interna:

- (1) Há sempre que considerar a eventualidade dos erros aleatórios de tipo I, mesmo dentro do limite de erro que os autores estavam dispostos a aceitar $(\alpha=0,05)$;

- (2) Para minimizar a probabilidade dos erros aleatórios de tipo II, calculou-se (através da ferramenta StatCalc inserido no programa estatístico Epi-Info $\left.^{\mathrm{TM}}\right)^{20}$ a dimensão da amostra necessária para se verificar a redução obtida da prevalência do alto risco cardiovascular de $38,58 \%$ para $24,37 \%$, com um $\alpha=0,05$ e um poder de $80 \%$, obtendo-se um valor estimado mínimo de 180 unidades amostrais (inferior à dimensão da população em estudo).

- (3) Consideram-se os possíveis erros sistemáticos, nomeadamente os seguintes vieses de informação:

- Viés de entrevistador - por ter havido um único observador.

Os dados registados foram baseados nos cuidados prestados por um Médico de Família recém-formado ainda com pouca experiência prática mas eventualmente mais sensibilizado para o problema das doen- ças cardiovasculares e predisposto para actuação mais «agressiva».

Os resultados obtidos podem advir da atitude do profissional perante o problema ou de outros factores relacionados com as características pessoais (personalidade, linguagem utilizada na sua relação com o paciente, alta motivação, etc.).

- Viés de medição - o facto de ter sido utilizado um esfigmomanómetro manual durante a maior parte do estudo pode ter condicionado uma menor precisão na avaliação e registo da TA, devido a provável tendência para o arredondamento dos valores obtidos, o que não acontece com o esfigmomanómetro automático utilizado nos últimos meses do estudo.

Não foram considerados os vieses de selecção, uma vez que o presente estudo analisou toda a população e não uma amostra da mesma.

Ao contrário dos erros sistemáticos, as variáveis de confundimento podem ser controladas pela análise estatística. Os autores analisaram as variáveis universais sexo e idade como possíveis variáveis de confundimento através da análise estratificada, tendo constatado uma melhoria significativa a nível do RCVG tanto no sexo masculino como no feminino, bem como nos idosos ( $\geq 65$ anos) e não idosos ( $<65$ anos).

Na interpretação dos resultados obtidos, é de salientar que a média dos valores do RCVG obteve uma redução maior que os FRCV individualmente. Tal se poderá dever ao facto de em cada doente ser mais importante o controlo de um dos FRCV em relação a outro para um 
mesmo nível (sinérgico) de RCVG.

Além disso, inicialmente, a população hipertensa encontrava-se globalmente enquadrada na categoria de alto risco (SCORE médio de 5,00), vindo posteriormente a evoluir para a categoria de baixo risco, ao reduzir-se o SCORE médio para 4,04.

Quanto aos FRCV major, verificou-se uma redução média da TA de $9,3 \%(-10,1 \mathrm{mmHg})$ para a TAS e um aumento de 3,2\% na TAD. Mas se compararmos os valores obtidos nos hipertensos de alto e baixo risco, verificaremos que a redução da TAS é de $-18,3 \mathrm{mmHg}$, ou seja, um valor muito aproximado daquele que representaria uma diminuição para metade da mortalidade por DIC e AVC (20mmHg) ao fim de 5 anos.

Verificou-se, todavia, um aumento ainda mais substancial de 133,3\% no grau de controlo tensional no final do estudo, o que corresponde uma evolução de $18,3 \%$ para $42,6 \%$ hipertensos controlados, valor que representa quase o quádruplo do obtido no estudo de Espiga de Macedo para Portugal $(11,2 \%)$.

No que diz respeito ao perfil lipídico, obteve-se uma redução média de $13,5 \mathrm{mg} / \mathrm{dl}(6,5 \%)$ para o CT, permanecendo 106 hipertensos (53,8\%) com CT $\geq 190 \mathrm{mg} / \mathrm{dl}$ ao fim de 3 anos de cuidados continuados, valor todavia inferior ao verificado em Portugal pelo estudo da Fundação Portuguesa de Cardiologia (68,5\%). Na análise do subgrupo de alto risco, verificou-se uma redução ainda mais acentuada de $11,2 \%$, o que ultrapassa os $10 \%$ necessários para condicionar uma redução de $25 \%$ na incidência de doença coronária aos 5 anos.

Quanto ao C-LDL, verificou-se uma redução média de 18,3mg/dl na população hipertensa e de $25,6 \mathrm{mg} / \mathrm{dl}$ no subgrupo de alto risco, o que, embora ainda não atingindo os valores de redução de $40 \mathrm{mg} / \mathrm{dl}$, que implicariam a redução de $23 \%$ na doença coronária, $19 \%$ de AVC e $21 \%$ de doenças cardiovasculares, permite sugerir que aos 5 anos de continuidade de cuidados se possam obter os valores necessários.

O presente estudo constitui um projecto-piloto para outras investigações de base populacional de maiores dimensões e com maior duração, que poderão confirmar a elevada redução no RCVG, através do controlo dos FRCV modificáveis potenciado pela continuidade de cuidados.

Em resumo, verificou-se, uma redução significativa do RCVG e uma melhoria dos FRCV major. Houve actuação adaptada aos níveis iniciais do risco, resultando em maior benefício naqueles pacientes com alto risco inicial.

\section{REFERÊNCIAS BIBLIOGRÁFICAS}

1. Direcção-Geral da Saúde. Programa Nacional de Prevenção e Controlo de Doenças Cardiovasculares. Circular Normativa n. ${ }^{\circ}$ 03/DSPCS, de 06/02/06. Lisboa: DGS; 2006.

2. Direcção de Serviços de Epidemiologia e Estatísticas da Saúde, Direcção-Geral da Saúde. Risco de morrer em Portugal: 1999-2005. Lisboa: DGS; 2006.

3. DIRECÇÃO-GERAL DA SAÚDE: Direcção-Geral da Saúde. Risco global cardiovascular. Circular Normativa n. ${ }^{\circ}$ 06/DSPCS, de 18/04/07. Lisboa: DGS; 2007.

4. Mafra F, Oliveira H. Avaliação do risco cardiovascular: metodologias e suas implicações na prática clínica. Rev Port Clin Geral 2008 Maio-Jun, 24 (3): 391-400.

5. Berlowitz DR, Ash AS, Hickey EC, Friedman RH, Glickman M, Kader B, et al. Inadequate management of blood pressure in a hypertensive population. N Engl J Med 1998 Dec 31, 339 (27): 1957-63.

6. Graham I, Atar D, Borch-Johnsen K, Boysen G, Burell G, Cifkova R, et al. European Guidelines on Cardiovascular Disease Prevention in Clinical Practice: Fourth Joint Task Force of the European Society of Cardiology. Eur J Cardiovasc Prev Rehabil 2007 Sep; 14 Suppl 2. S1-113.

7. Macedo ME, Lima MJ, Silva AO, Alcântara P, Ramalhinho V, Carmona J Prevalence, awareness, treatment and control of hypertension in Portugal: the PAP Study. J Hypertens 2005 Sep; 23 (9): 1661-6.

8. Lewington S, Clarke R, Qizilbach N, Peto R, Collins R. Age-specific relevance of usual blood pressure to vascular mortality: a meta-analysis of individual data for one million adults in 61 prospective studies. Lancet 2002 dec 14; 360 (9349): 1903-13.

9. Direcção-Geral da Saúde. Plano Nacional de Saúde 2004-2010. Lisboa: DGS; 2003.

10. Baigent C, Keech A, Kearney PM, Blackwell L, Buck G, Pollicino C, et al; Cholesterol Treatment Trialists (CTT) Collaborators. Efficacy and safety of cholesterol-lowering treatment: prospective meta-analysis of data from 90.056 participants in 14 randomised trials of statins. Lancet 2005 Oct 8; 366 (9493): 1267-78.

11. Instituto Nacional de Saúde Dr. Ricardo Jorge. $3^{\circ}$ Inquérito Nacional de Saúde 1998/1999. Disponível em: http://www.insarj.pt [acedido em 28/10/2008].

12. Instituto Nacional de Saúde Dr. Ricardo Jorge. $4^{\circ}$ Inquérito Nacional de Saúde 2005/2006. Disponível em: http://www.insarj.pt [acedido em 28/10/2008].

13. Cabana MD, Jee SH. Does continuity of care improve patient outcomes? J Fam Pract 2004 Dec; 53 (12): 974-80.

14. Saultz JW, Lochner J. Interpersonal continuity of care and care outcomes: a critical review. Ann Fam Med 2005 Mar-Apr; 3 (2): 159-66 .

15. Chobanian AV, Bakris GL, Black HR, Cushman WC, Green LA, Izzo JL Jr, et al. The Seventh Report of the Joint National Committee on Prevention, Detection, Evaluation, and Treatment of High Blood Pressure: The 
JNC 7 Report. JAMA 2003 May 21; 289 (19): 2560-72.

16. DIRECÇÃO-GERAL DA SAÚDE: Diagnóstico, Tratamento e Controlo da Hipertensão Arterial. Circular Normativa n. ${ }^{\circ}$ 02/DGCG, de 31/03/04. Lisboa: DGS; 2004.

17. European Society of Cardiology. Disponível em: http://www.escardio.org/ knowledge/decision_tools [acedido em 28/10/2008].

18. Excel ${ }^{\circledR}$ versão 2003 (Windows). Redmond, VA: Microsoft Corp.; 2003.

19. Norusis MJ SPSS ${ }^{\circledR} / P C+$. Advanced Statistics, versão 10.0. Chicago: SPSS Inc; 1999.

20. Epi-InfoTM versão 3.5.1. Atlanta, GA: Centers for Disease Control and Prevention (CDC); 2008. Disponível em: http://www.cdc.gov/epiinfo/ [acedido em 28/10/2008].

\author{
ENDEREÇO PARA CORRESPONDÊNCIA \\ Hiroshi Okai \\ Rua do Agueiro, 256-1 Esq. \\ Mafamude, 4400-004 Vila Nova de Gaia \\ Tel./Fax: 256922341 \\ E-mail: hiroshi.okai@gmail.com \\ Recebido em 20/11/2008 \\ Aceite para publicação em 02/03/2009
}

\begin{abstract}
Introduction: Cardiovascular diseases are the main causes of death in Portugal, as it represents 34\% of deaths occurred in 2005. The National Health Department recommends using the table derived from the project SCORE in order to calculate the global cardiovascular risk (GCVR), which allows us to identify patients with a higher risk who benefit the most with the effective control of cardiovascular risk factors (CVRF).

The Family Doctor is in the appropriate position to act out on modifiable CVRF of the hypertensive population and hence reduce their GCVR. There are several evidences demonstrating that continuity of care results in health improvement obtained in various chronic conditions, such as hypertension, diabetes mellitus and asthma.

Goals: Verify if continuity of care provided to the hypertensive patients by the Family Doctor influence: (1) reduction of GCVR; (2) improvement of major CVRF; (3) higher reduction of blood pressure and lipid profile of the hypertensive patients with an initially high GCVR.

Methodology: Quasi-experimental retrospective longitudinal study. Target Population: Hypertensive patients diagnosed until 2004 and usual attendants of the hypertension appointments between 2004 and $2007(n=197)$. Studied variables: BP, lipid profile, smoking and GCVR.

Results: Comparing the average values of the GCVR obtained with the SCORE table a significant reduction of $19,2 \%$ was obtained during 3 years. Concerning CVRF, we verified: a significant average reduction of $9,3 \%$ at the level of systolic blood pressure (SBP) and an increase of 3,2\% at the diastolic blood pressure (DBP); a significant average reduction of $6,5 \%$ and $13,8 \%$ for TC and LDL-cholesterol (LDL-C); a significant increase of HDL-cholesterol (HDL-C) and the triglycerides (TC) of 5,4\% and 8,4\%, correspondently; and a significant reduction of $50 \%$ of smoking status. The hypertensive patients who were initially considered higher risk, obtained a three times reduction of the SBP and TC and a two times reduction of the LDL-C compared to those patients of lower risk.

Conclusions: A significant reduction of GCVR and an improvement of major CVRF were verified. There was an adapted performance to the initial levels of risk; which resulted in a great benefit to those with high initial risk.
\end{abstract}

Keywords: Global Cardiovascular Risk; Continuity of Care; Family Doctor. 